\title{
Customized Sequential Quadratic Programming for Solving Large-Scale AC Optimal Power Flow
}

This paper was downloaded from TechRxiv (https://www.techrxiv.org).

\section{LICENSE}

CC BY 4.0

SUBMISSION DATE / POSTED DATE

06-10-2021 / 12-10-2021

\section{CITATION}

Sadat, Sayed Abdullah; Sahraei-Ardakani, mostafa (2021): Customized Sequential Quadratic Programming for Solving Large-Scale AC Optimal Power Flow. TechRxiv. Preprint.

https://doi.org/10.36227/techrxiv.16748206.v1

$\mathrm{DOI}$

10.36227/techrxiv.16748206.v1 


\section{Customized Sequential Quadratic Programming for Solving Large-Scale AC Optimal Power Flow}

\author{
Sayed Abdullah Sadat \\ Department of Electrical and Computer Engineering \\ University of Utah \\ Salt Lake City, United States \\ sayed_abdullah@ieee.org
}

\author{
Mostafa Sahraei-Ardakani \\ Department of Electrical and Computer Engineering \\ University of Utah \\ Salt Lake City, United States \\ mostafa.ardakani@utah.edu
}

\begin{abstract}
After decades of research, efficient computation of AC Optimal Power Flow (ACOPF) still remains a challenge. ACOPF is a nonlinear nonconvex problem, and operators would need to solve ACOPF for large networks in almost real-time. Sequential Quadratic Programming (SQP) is one of the powerful second-order methods for solving large-scale nonlinear optimization problems and is a suitable approach for solving ACOPF with large-scale real-world transmission networks. However, SQP, in its general form, is still unable to solve large-scale problems within industry time limits. This paper presents a customized Sequential Quadratic Programming (CSQP) algorithm, taking advantage of physical properties of the ACOPF problem and the choice of the best performing ACOPF formulation. The numerical experiments suggest that CSQP outperforms commercial and noncommercial nonlinear solvers and solves test cases within the industry time limits. A wide range of test cases, ranging from 500-bus systems to 30,000 -bus systems, are used to verify the test results.

Index Terms-AC optimal power flow (ACOPF), large-scale networks, nonlinear programming, power system operation, sequential quadratic programming (SQP).
\end{abstract}

\section{INTRODUCTION}

$\mathbf{P}$ OWER system operation aims to deliver power to customers in a reliable and cost-effective manner. Optimal and reliable power dispatch is an optimization problem, which in its original form is referred to as the "alternating current optimal power flow" (ACOPF) problem [1], [2]. ACOPF is a nonlinear optimization problem with non-convex constraints, which makes it NP-hard [3], [4].

The computational challenge is also significant because of the large size of real-world networks. The power grid in the United States is the largest machine ever built by humans, with hundreds of thousands of buses, transmission elements, and generators in three synchronous interconnections. According to the U.S. Energy Information Administration, the aggregate sum of annual retail transactions in the US power grid now exceeds USD 400 billion [5]. Clearly, a cost-effective operation is important due to the economic impacts of this crucial infrastructure.

Because of the difficulty of solving the ACOPF problem, the majority of the grid operators use some form of a direct current optimal power flow (DCOPF) formulation [6]. The DCOPF linearization used in power system operation is based on a few assumptions that help convert the nonlinear ACOPF problem to a linear formulation [7], [8] by applying rough approximations of voltages and reactive power flows.

Although the approximations enable solving optimal power flow within the practical time constraints, it is mathematically proven that the solutions based on these approximations are never feasible [9]. Therefore, it requires the application of conservative transmission limits to keep sufficient margins to achieve feasibility around the DCOPF solution. This most likely results in an inefficient and sub-optimal operation of the grid. In the United States, the economic costs represent tens of billions of dollars each year. Moreover, a good solution to ACOPF is critical to many complex decision-making processes, including consideration of trade-offs between active and reactive power flows [10], [11]. Thus, developing a method that efficiently solves the ACOPF problem can have a significant economic impact. Over the past few decades, many methods have been proposed to solve the ACOPF problems. However, the majority of them overlook the impact of formulation on the performance of ACOPF solution, especially when solving large-scale problems. Many methods have been proposed and tested for solving ACOPF, including a variety of convex relaxation techniques [12]-[15]. Another approach that has recently attracted growing interest among researchers and power engineers, and has the potential to perform well is the sequential linear programming (SLP) method for solving different ACOPF formulations [16], [17]. This method takes advantage of the linear representation of network flows which provides the capability of easy integration into the exiting energy management system (EMS) and leverages the deployment of off-the-shelf commercial linear programming solvers [10]. It has also been shown that the performance of SLP, in terms of convergence quality, objective value, and computational performance, largely depends on the initialization of the SLP algorithm [18], [19].

Anya Castillo et al. in [10] apply Successive Linear Programming (i.e., Sequential Linear Programming) to solve the AC Optimal Power flow problem. The paper advantages current voltage in rectangular form over other formulations of ACOPF and adopts a heuristic approach for controlling the step size. The main focus of the algorithm introduced in [10] is to obtain an AC feasible solution to the original ACOPF problem. Z. Yang et al in [20] presents a comparison of 
different linearization techniques for the constraints in ACOPF that contains nonlinear terms. The linearization range from DC model linearization to first-order Taylor series approximation and preservation of second-order terms. The comparison demonstrates the impact of different linearization techniques on the accuracy of linearization of the original ACOPF model in canonical form. Z. Yang et al. in [21], [22] introduce an iterative approach to solve ACOPF problem. Their method, similar to the work in [20], is based on the polar form of state variables. The work is focused on obtaining a feasible solution and uses constraints reduction techniques similar to [10]. The linearization techniques mentioned in [21] with error handling approach and better computation time is what makes this work distinctive compared to the work in [10], though it does not present any step control algorithm. P. Fortenbacher and T. Demiray in [23] present a non-iterative linear (LP)/quadratic programming (QP) based solution approach for an optimal power flow problem. The paper focuses on modeling absolute loss approximations and adding it as linear constraints to the ACOPF problem. In one of its approaches it also proposes using integer variables for modeling losses, which becomes computationally intractable for large networks.

A. Mohamed and B. Venkatesh in [24] present a line-wise optimal power flow formulation solved using successive linear programming method. The authors claim better performance of their proposed method over MATPOWER solver and bus-wise ACOPF formulation. The formulation uses square of nodal voltage magnitude as control variable. The work solves a set of nonlinear equations using the Newton-Raphson method before solving each linear programming subproblem. The work also suggests the choice of step-size based on experience with each network. L. Sampath et al. in their in [25] propose solving ACOPF problem in polar form using a trust-region sequential linear programming method. The method adapts the first order optimality condition from necessary KarushKuhn-Tucker (KKT) conditions proposed in [26], and therefore, local optimality is sought by the algorithm. The work also uses a merit function approach to decide whether to accept or reject a step during the iterative solution of the trust-region LP subproblem. Finally, in [27], an SLP method is proposed consisting of a sequence of constructed supporting hyperplanes and halfspaces to obtain a performance comparable with IPM, but for smaller cases (i.e., < 4000-bus systems).

The past work presents a great deal of progress in solving ACOPF problems by presenting different choices for the selection of an efficient algorithm. However, no existing algorithm can solve ACOPF for large-scale problems in almost real time (i.e., $<5$-minutes).

The choice of SQP method for solving ACOPF problems has many advantages and disadvantages. The advantages of SQP algorithm include the following: a) Unlike interior point methods (IPMs), SLP method is more sensitive to initialization and therefore, more appropriate and computationally efficient for time-series solution of ACOPF [18], [19]; b) SQP methods may leverage commercial LP solvers which are remarkably fast and are reliable [27]; c) Locational Marginal Prices
(LMP), which are the linchpin of electricity markets, are easier to obtain using LP; d) The system operators and the existing energy management systems (EMS) prefer linearized methods and therefore, SQP can easily integrate with existing EMS systems [6], [28], to name a few. The downsides of the SQP method is that it is much slower compared to the IPM method for large scale problems and the calculation of Hessian matrix can become challenging.

The aim of this paper is to develop a customized SQP algorithm for solving industry-grade large scale ACOPF problems by addressing the challenges of the original SQP algorithm by taking advantage of the physical properties and structures of the ACOPF while leverage existing EMS tools and commercial optimization solvers. The contribution of this paper can be summarized as follows:

1) Implementation of approximated active set approach for SQP method.

2) Improving the performance of SQP method by replacing addressing the computational bottlenecks.

3) Finally, customizing SQP method for faster convergence to solve large-scale ACOPF problems within the industry time limits.

The rest of the paper is organized as follows. In Section II, optimal power flow and the best performing ACOPF formulation are introduced. In section III, customized SQP is presented. In section IV, the results of numerical experiments are presented. Finally, in section $\mathrm{V}$ the paper is concluded.

\section{Optimal Power Flow (OPF)}

An optimal power flow constitutes the core of power system operations. It is an optimization extension of the power flow analysis [29], where in addition to feasibility, an optimal dispatch is also desired. The objective of power system operation is to serve the customers at the minimum cost, taking into consideration the physical constraints of the system. This can be expressed as an optimization problem, referred to as optimal power flow (OPF). In addition to power system operations, a solution to the optimal power flow is also essential for power system planning. In an ACOPF problem, there are different approaches to model a transmission branch in a power system. The most commonly used representation of the transmission branch is called the $\pi$-model, which is applied in this paper. We use the conventions and formulations derived in [30], [31].

An ACOPF problem can be formulated as an optimization problem as shown in (1).

$$
\begin{aligned}
& \min \sum_{g \in \mathcal{G}} c_{2 g} \cdot p_{g}^{2}+c_{1 g} \cdot p_{g}+c_{0 g} \\
& \text { s.t. } \quad \sum_{g \in \mathcal{G}_{n}} p_{g}-\sum_{d \in \mathcal{D}_{n}} p_{d}=\sum_{k \in \mathcal{N}} V_{n} \cdot V_{k} \cdot\left(G_{n k} \cdot \cos \theta_{n k}\right. \\
& \left.+B_{n k} \cdot \sin \theta_{n k}\right)+G_{n}^{L} \cdot V_{n}^{2}, \forall n \in \mathcal{N} . \\
& \sum_{g \in \mathcal{G}_{n}} q_{g}-\sum_{d \in \mathcal{D}_{n}} q_{d}=\sum_{k \in \mathcal{N}} V_{n} \cdot V_{k} \cdot\left(G_{n k} \cdot \sin \theta_{n k}\right. \\
& \left.-B_{n k} \cdot \cos \theta_{n k}\right)-B_{n}^{L} \cdot V_{n}^{2}, \forall n \in \mathcal{N} . \quad(1 \mathrm{c}) \\
& 2 \cdot\left|y_{i j}^{n e t}\right| \cdot\left|Y_{i j}\right| \cdot V_{i} \cdot V_{j} \cdot \cos \left(\theta_{n k}+\theta_{n k}^{y Y}\right)+\left|Y_{i j}\right|^{2} \cdot V_{j}^{2}
\end{aligned}
$$




$$
\begin{aligned}
&+\left|y_{i j}^{\text {net }}\right|^{2} \cdot V_{i}^{2} \leq\left(I_{i j}^{\text {Max }}\right)^{2}, \forall\{i, j\} \in 2 \mathcal{K} . \\
& V_{n}^{\text {Min }} \leq V_{n} \leq V_{n}^{\text {Max }}, \forall n \in \mathcal{N} . \\
& p_{g}^{\text {Min }} \leq p_{g} \leq p_{g}^{\text {Max }}, \forall g \in \mathcal{G} . \\
& q_{g}^{\text {Min }} \leq q_{g} \leq q_{g}^{\text {Max }}, \forall g \in \mathcal{G} .
\end{aligned}
$$

Equation (1) represents one of the general representations of an ACOPF problem in its complex form based on the grid model presented in [32]. $c_{2 g}, c_{1 g}$, and $c_{0 g}$ are the coefficients of quadratic cost function of generator $g, s_{g}=p_{g}+j q_{g}$ is the apparent power output of generator $g, v_{n}=v_{n}^{r}+j v_{n}^{i}=V_{n} e^{j \theta_{n}}$ is the voltage at bus $n, s_{d}=p_{d}+j q_{d}$ is the complex load, $V_{n}^{M i n}$ and $V_{n}^{M a x}$ are lower and upper voltage bounds at bus $n, I_{i j}^{M a x}$ is the current thermal limit of line $\{i, j\}$, $s_{g}^{M i n}=p_{g}^{M i n}+j q_{g}^{M i n}, s_{g}^{M a x}=p_{g}^{M a x}+j q_{g}^{M a x}$ are the complex generator $g$ bounds, and $Y_{n}^{L}=G_{n}^{L}-j B_{n}^{L}$ is the complex Shunt element connected to bus $n . \mathcal{N}$ is the set of buses, $\mathcal{G}$ is the set of generators, and $\mathcal{K}$ is the set of branches in the network.

The ACOPF shown in (1) is a nonlinear nonconvex problem with quadratic objective function. The quadratic objective function (2a) can be linearized using (2) as described in [32]

$$
\begin{array}{lll}
\min & \sum_{g \in \mathcal{G}} c_{g} & \\
\text { s.t. } & c_{g}=\sum_{h \in \mathcal{H}_{g}} c_{g h} t_{g h}, & \forall g \in \mathcal{G} . \\
& \sum_{h \in \mathcal{H}_{g}} p_{g h} t_{g h}=p_{g}, & \forall g \in \mathcal{G} . \\
& t_{g h} \geq 0, & \forall g \in \mathcal{G} . \\
& \sum_{h \in \mathcal{H}_{g}} t_{g h}=1, & \\
\text { III. Customized SEQUENTIAL QuAdRATIC } &
\end{array}
$$

\section{Customized Sequential Quadratic PROGRAMMING}

SQP is an optimization method for solving a nonlinear optimization problems, which is based on iteratively solving second order Taylor Series approximation of the nonlinear problem. The algorithm is initialized with an estimate solution. Then, the algorithm recursively solves linearly constraint quadratic optimization subproblem and updates the operation point until it satisfies a predefined convergence criteria. The approach has almost all advantages of SLP. In addition, since SQP is second-order method, it offers faster and guaranteed convergence [33] compared to SLP. Similar to SLP the approach can leverage off-the-shelve commercial linear solvers which can efficiently solve the LP subproblem and It can also be readily extended to more complex decision-making processes in power systems [7], [11]. It takes into account even the weak couple between real and reactive powers, which makes it suitable for even highly congested and higher reactive to real load ratio.

Let's assume eq. (3) represents a nonlinear problem.

$$
\begin{array}{ll}
\min _{x} & f(x) \\
\text { s.t. } & c_{i}(x)=0, i \in \mathcal{E}
\end{array}
$$

$$
c_{i}(x) \geq 0, \quad i \in \mathcal{I}
$$

The quadratic subproblem can be mathematically expressed as

$$
\begin{array}{ll}
\min _{P} & f_{k}+\nabla f_{k}^{T} \times p+\frac{1}{2} p^{T} \nabla_{x x}^{2} \mathcal{L}_{k} p+\mu_{p} \sum_{i \in \mathcal{E}}\left(v_{i}+w_{i}\right) \\
& +\mu_{p} \sum_{i \in \mathcal{I}}\left(t_{i}\right) \\
\text { s.t. } & \nabla c_{i}\left(x_{k}\right)^{T} \times p+c_{i}\left(x_{k}\right)=v_{i}-w_{i}, \quad i \in \mathcal{E} \\
& \nabla c_{i}\left(x_{k}\right)^{T} \times p+c_{i}\left(x_{k}\right) \geq-t_{i}, \\
& v, w, t \geq 0
\end{array}
$$

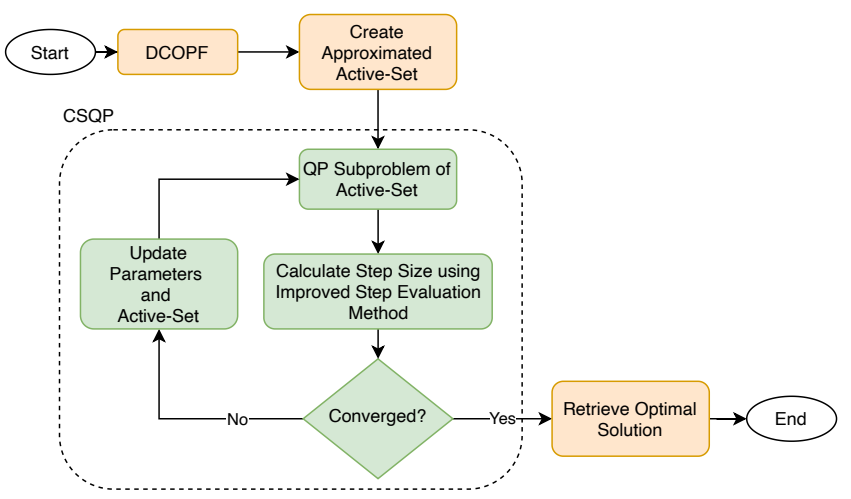

Fig. 1: Flowchart of the SCQP algorithm

To improve the performance of the general SQP method we introduce a customized SQP algorithm described in fig. 1 . The method takes advantage of optimization structure specific to ACOPF. It also utilizes available tools at the operating centers, such DCOPF, etc.

\section{A. DCOPF}

DCOPF is a linearized approximation of the original ACOPF problem (1). DCOPF is based on a number of assumptions that approximates the ACOPF problem to an LP problem [6]. In this paper, we utilize DCOPF for two main purposes, namely, initialization and defining an approximate active-set for the CSQP algorithm.

1) CSQP Initialization: SQP method is sensitive to initialization and the existing EMS system has the capability to solve DCOPF. Hence, we use DCOPF solution to initialize the proposed algorithm. An appropriate initialization with topology information can significantly reduce the total iteration numbers necessary for the problem to converge [34]. The advantage of this approach is that it provide a warm-start solution for the initialization of the SQP method.

2) Approximated Active-Set: The active set $A(x)$ at any feasible $x$ consists of the equality constraint indices from $\mathcal{E}$ together with the indices of the inequality constraints $i$ for which $c_{i}(x)=0$; that is,

$$
\mathcal{A}(x)=\mathcal{E} \cup\left\{i \in \mathcal{I} \mid c_{i}(x)=0\right\} .
$$


At a feasible point $x$, the inequality constraint $i \in \mathcal{I}$ is said to be active if $c_{i}(x)=0$ and inactive if the strict inequality $c_{i}(x)>0$ is satisfied. In the approximated activeset approach we try to obtain an approximation of the $\mathcal{A}(x)$ using the DCOPF solution, notated as $\mathcal{A}^{\prime}(x)$ and solve the QP subproblem (4) only for the $\mathcal{A}^{\prime}(x)$. This can significantly reduce the computation complexity of the QP subproblems for a large-scale problem.

\section{B. Regulators}

One of the computational challenges of SQP method is the calculation of hessian in the objective function of the QP subproblem (4a). However, in ACOPF there is a relationship between control and state variables. Therefore, using control variables helps in captures the approximately curvature otherwise captured by the hessian term while saving a significant portion of computation in the CSQP algorithm. The quadratic term in the objective using control variables also constitutes regulators which are:

$$
\mu_{p} \cdot p_{p g}^{T} \cdot p_{p g}+\mu_{q} \cdot q_{p g}^{T} \cdot q_{p g}
$$

where $\mu_{p}$ and $\mu_{q}$ are the weights used to appropriate the regulator and helps in replicating the impact of the hessian term (i.e., $\frac{1}{2} p^{T} \nabla_{x x}^{2} \mathcal{L}_{k} p$ ) in the original SQP method. $p_{p g}$ and $q_{p g}$ are the direction variables corresponding to generator real and reactive power variables. However, this approximation comes at a cost, where we lose the convergence guarantee otherwise warranted for the general SQP method under certain conditions described in [33].

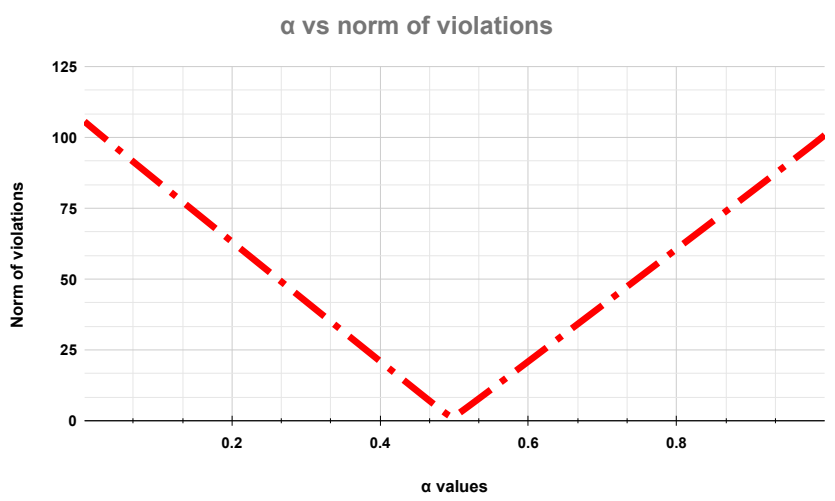

Fig. 2: A plot of $\alpha$ vs $\left\|c_{i}\right\|_{2}$ for a 30,000-bus system.

\section{Customized Violations Evaluation:}

The original SQP line search algorithm as described in [33], evaluates the violation using the direction obtained for each variable in the QP subproblem (4). The convergence can be significantly improved by once again utilizing the physical behaviour of the ACOPF. The variables in ACOPF can be divided into control and state variables. Physically, the change in control variables results in the changes in the state variables. Therefore, instead of evaluating the violation using the direction obtained for control variables from the QP subproblem, we use the available capacity for them. Additionally, the violations can be only checked for constraints with nonlinear terms. This modification will need an extra optimization step at the end to retrieve the optimal values of the linear constraints. The customization significantly improves the convergence speed of the SQP algorithm.

\section{Improvement of step evaluation}

During the search for step size in SQP line search algorithm, there exists the lowest value of norm of violations corresponding to an $\alpha$ value between 1 and 0 . Finding this value helps significantly in speeding up the convergence of the sequential quadratic programming algorithm instead of choosing the largest step size that can give satisfy the merit function described in (7).

$$
\phi\left(x_{k}+\alpha_{k} P_{k} ; \mu_{k}\right) \leq \phi\left(x_{k}, \mu_{k}\right)+\eta \alpha_{k} D\left(\phi\left(x_{k} ; \mu_{k}\right) ; p_{k}\right)
$$

where $\phi\left(x_{k}, \mu_{k}\right)=f(x)+\mu\left\|c_{k}\right\|_{1}, D\left(\phi\left(x_{k} ; \mu_{k}\right) ; p_{k}\right)=$ $\nabla f_{k}^{T} P_{k}-\mu\left\|c_{k}\right\|_{1}$, and $\eta \in(0,0.5)$.

Fig. 2 shows the plot of the $\alpha$ vs $\left\|c_{i}\right\|_{2}$ for an ACOPF problem of a 30,000 bus size. The pattern in this problem as well as other synthetic networks show that the choice of $\alpha$ value makes a significant difference. For instance, in this example $\alpha=1$ or $\alpha=0.001$ have norm of violations greater than 100 , whereas at $\alpha=0.497$ the norm violations is 1.4 . The is a significant change in norm of violations.

\section{NUMERICAL EXPERIMENTS}

Various numerical experiments were performed on an 8-core Intel(R) i9-9980 HK CPU @ 2.40 GHz with 32 GB RAM system to test and validate the performance of different ACOPF formulations. The programming for numerical experiments are implemented in Julia v1.5.3 using IPOPT v3.13.2, KNITRO 12.4, and JuMP v0.21.5. in a Linux Ubuntu operating system.

The numerical experiments were performed on the U.S. Department of Energy's Grid Optimization Competition Challenge I test cases ranging from 500-bus to 30,000-bus systems [35].

Fig. 3 compares the performance of popular nonlinear solvers such as IPOPT with two linear solvers and commercial solver such as KNITRO for all the test cases mentioned above. For IPOPT solver we use the default solver as well the as the best performing linear solver for ACOPF problems [31]. The setting for KNITRO linear solver is set at 0, allowing KNITRO to choose its linear solver. It can be observed that CSQP algorithm has shown consistently superior performance, solving all test cases in less than two minutes. The results of SQP algorithm implemented by KNITRO is excluded to avoid the disturbance of visualization of results from other algorithms as SQP algorithm took more than an hour for largescale problems.

Fig. 4 show the histogram plot of the percentage of optimality gap from local minima for all test cases using CSQP algorithm. The plot shows that about $85 \%$ of the test cases had $0.25 \%$ to $0 \%$ optimality gap. The largest optimality gap is less than $0.7 \%$. 


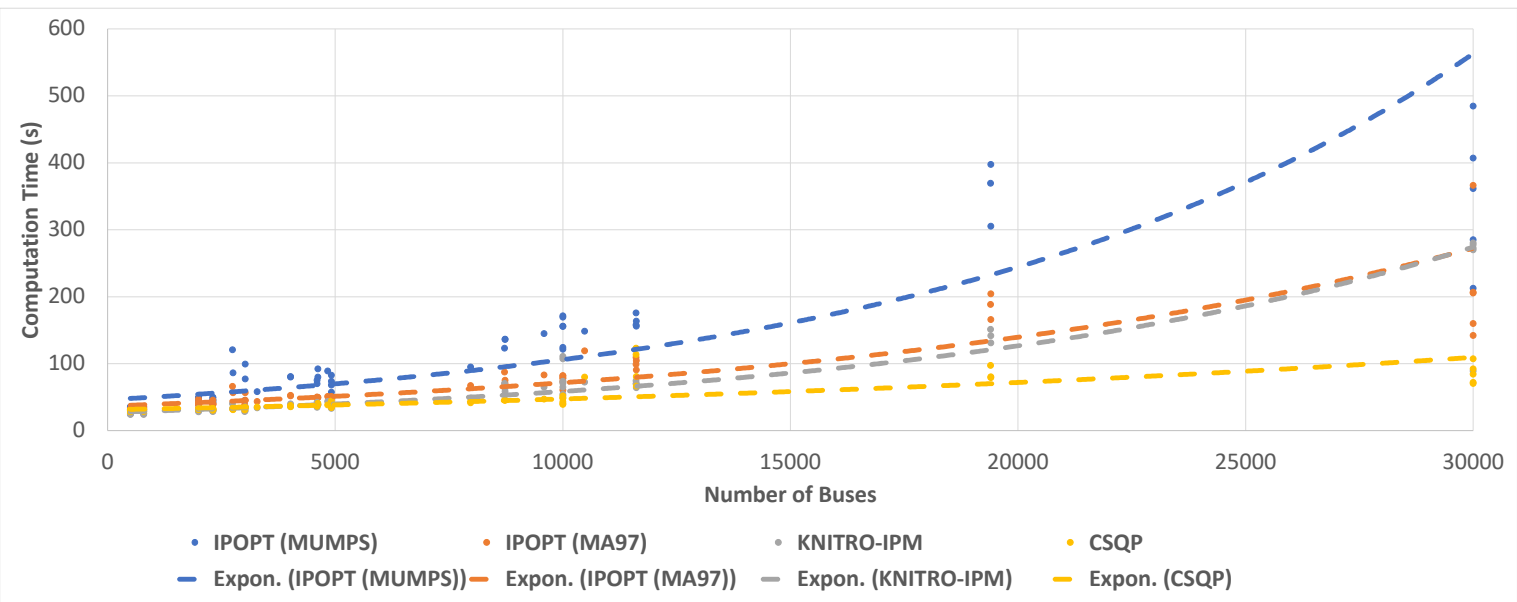

Fig. 3: Comparison of the computation time of IPOPT with MUMPS linear solver, IPOPT with MA97 linear solver, KNITRO interior point method (IPM), and Customized Sequential Quadratic Programming (CSQP) for a wide range of test cases ranging from 500-bus to 30,000-bus systems.

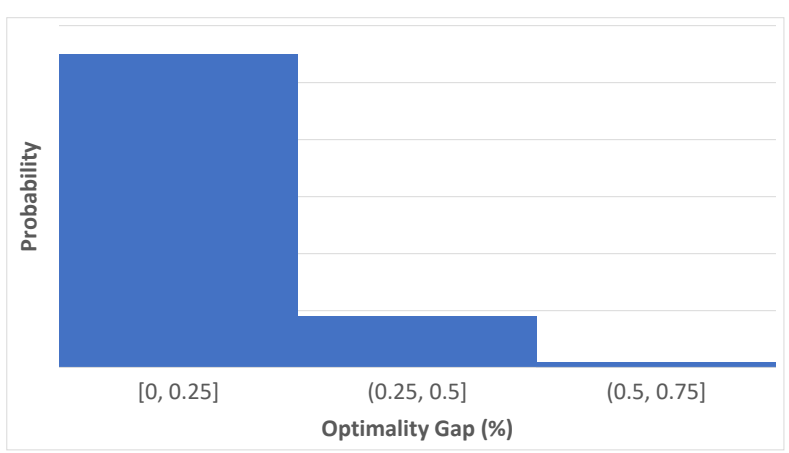

Fig. 4: A histogram plot of CSQP solution gap (\%) from local optimality for all the test cases.

\section{Performance Profile}

Finally, To assess the performance of different algorithms, we plot the performance profile [30], [36] using different scenarios of a wide range of test cases described in [35].

Let $\mathcal{A L}$ be the set of different algorithms and solvers. For each problem $p \in \mathcal{P}$ and algorithm $a \in \mathcal{A L}$, let $t_{p a}$ be the solution time for solving problem $p$ using algorithm $a$. We define the performance ratio

$$
r_{p a}:=\frac{t_{p a}}{\min _{a \in \mathcal{A L}} t_{p a}}
$$

which represents the performance of algorithm $a$ as compared with the best performance by any algorithm on problem $p$. Now we define the performance of algorithm $a$ on any given problem as the probability for algorithm $a$ that a performance ratio $r_{p a}$ is within a factor $\tau$ of the best possible ratio; that is,

$$
\rho_{a}(\tau):=\frac{1}{|\mathcal{P}|}\left|\left\{p \in \mathcal{P}: r_{p a} \leq \tau\right\}\right|
$$

Fig. 5 shows the performance profile of different algorithms such as IPOPT (MUMPS), IPOPT (MA97), KNITRO-IPM,
KNITRO-SQP, and CSQP on a wide range of test cases mentioned before using (9). The proposed CSQP algorithm outperforms all other algorithms and solvers including the commercial solver KNITRO. CSQP algorithm is able to solve $100 \%$ of test cases at $\tau=2$. Whereas, KNITRO-IPM at $\tau=4$, IPOPT (MA97) at $\tau=4.5$, and IPOPT (MUMPS) at $\tau=6$. KNITRO-SQP is not able to solve even $20 \%$ of test cases at $\tau=6$. The plot shows that the proposed customization to the SQP method improves the performance of the SQP from the worst performing algorithm to outperforming best nonlinear programming algorithms. The significant computation comes at a minimal trade off in optimality as shown in fig. 4.

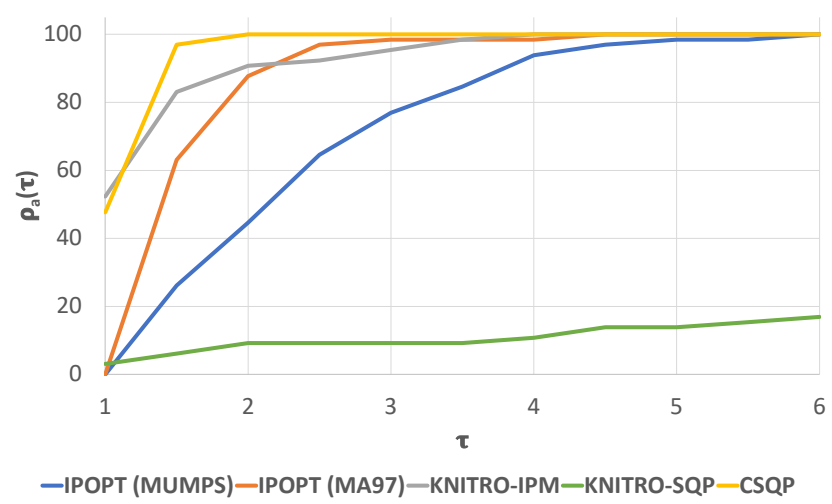

Fig. 5: Performance profile of IPOPT (MUMPS), IPOPT (MA97), KNITRO-IPM, KNITRO-SQP, and CSQP using a wide range of networks ranging from 500-bus to 30,000-bus systems.

\section{Conclusion}

The paper presents a customized sequential quadratic programming algorithm for solving ACOPF problem. The paper chooses the best performing ACOPF formulation, based on a 
previous ACOPF formulation analysis. The paper introduces approximated active-set for solving ACOPF, which reduces the computation complexity of the QP sunproblems. It also replaces the Hessian term of the QP subproblem with regulators, thereby taking advantage of the physical property of ACOPF. Furthermore, a customized violation evaluation is introduced that helps in much faster convergence of the algorithm. Finally, an improved step size evaluation method is proposed, which further speeds up the CSQP algorithm. The results show that the proposed algorithm solves all U.S. Department of Energy's Grid Optimization competition Challenge I test cases in less than two minutes. Numerical experiments were performed to compare the performance of CSQP with other commercial and popular solvers. The results confirms the superiority of CSQP for solving ACOPF over other solvers. The results also show that the proposed customization upgrades the SQP method from the worse performing algorithm to the best performing algorithm. These improvements are comes at a negligible cost, which is a small optimality gap for some of the test cases. In the future, we plant to investigate appropriate weights for the regulators that may lead to practically zero optimality gaps for all test cases.

\section{REFERENCES}

[1] M. B. Cain, R. P. O'neill, A. Castillo et al., "History of optimal power flow and formulations," Federal Energy Regulatory Commission, vol. 1, pp. 1-36, 2012.

[2] S. A. Sadat, D. Haralson, and M. Sahraei-Ardakani, "Security versus computation time in iv-acopf with socp initialization," in 2018 IEEE International Conference on Probabilistic Methods Applied to Power Systems (PMAPS), 2018, pp. 1-6.

[3] A. Castillo and R. P. O'Neill, "Computational performance of solution techniques applied to the acopf," Federal Energy Regulatory Commission, Optimal Power Flow Paper, vol. 5, 2013.

[4] D. K. Molzahn and I. A. Hiskens, A Survey of Relaxations and Approximations of the Power Flow Equations, 2019, vol. 4, no. 1-2. [Online]. Available: http://dx.doi.org/10.1561/3100000012

[5] Energy Information Administration, "Revenue from sales of electricity to ultimate customers," 2019. [Online]. Available: https://www.eia.gov/ electricity/annual/html/epa_02_03.html

[6] B. Stott, J. Jardim, and O. Alsaç, "Dc power flow revisited," IEEE Transactions on Power Systems, vol. 24, no. 3, pp. 1290-1300, 2009.

[7] S. A. Sadat and L. Fan, "Mixed integer linear programming formulation for chance constrained mathematical programs with equilibrium constraints," in 2017 IEEE Power Energy Society General Meeting, 2017, pp. $1-5$.

[8] S. A. Sadat, "Optimal bidding strategy for a strategic power producer using mixed integer programming," 2017.

[9] K. Baker, "Solutions of DC OPF are Never AC Feasible," preprint, 2020.

[10] A. Castillo, P. Lipka, J.-P. Watson, S. S. Oren, and R. P. O’Neill, "A successive linear programming approach to solving the iv-acopf," IEEE Transactions on Power Systems, vol. 31, no. 4, pp. 2752-2763, 2015.

[11] S. A. Sadat and M. Sahraei-Ardakani, "Cascading failure mitigation via transmission switching," preprint, 2021.

[12] J. Lavaei and S. H. Low, "Zero duality gap in optimal power flow problem," IEEE Transactions on Power Systems, vol. 27, no. 1, pp. 92-107, 2012.

[13] R. Madani, S. Sojoudi, and J. Lavaei, "Convex relaxation for optimal power flow problem: Mesh networks," IEEE Transactions on Power Systems, vol. 30, no. 1, pp. 199-211, 2015.

[14] S. H. Low, "Convex relaxation of optimal power flow-part i: Formulations and equivalence," IEEE Transactions on Control of Network Systems, vol. 1, no. 1, pp. 15-27, 2014.

[15] H. Hijazi, C. Coffrin, and P. Van Hentenryck, "Convex quadratic relaxations for mixed-integer nonlinear programs in power systems," Mathematical Programming Computation, vol. 9, no. 3, pp. 321-367, 2017.
[16] R. P. O'Neill, A. Castillo, and M. B. Cain, "The IV formulation and linear approximations of the ac optimal power flow problem," FERC, 2012.

[17] S. A. Sadat and M. Sahraei-Ardakani, "Tuning Successive Linear Programming to Solve AC Optimal Power Flow Problem for Large Networks ," preprint, 2021.

[18] S. A. Sadat, D. Haralson, and M. Sahraei-Ardakani, "Evaluation of various techniques to warm-start a successive linear programming algorithm for solving the iv acopf," in 2018 IEEE Power Energy Society General Meeting (PESGM), 2018, pp. 1-5.

[19] S. A. Sadat and M. Sahraei-Ardakani, "Initializing Successive Linear Programming Solver for ACOPF using Machine Learning," in 2020 IEEE North American Power Symposium (NAPS), 2020, pp. 1-6.

[20] Z. Yang, H. Zhong, Q. Xia, and C. Kang, "Solving opf using linear approximations: fundamental analysis and numerical demonstration," IET Generation, Transmission Distribution, vol. 11, no. 17, pp. 41154125, 2017.

[21] Z. Yang, H. Zhong, Q. Xia, A. Bose, and C. Kang, "Optimal power flow based on successive linear approximation of power flow equations," IET Generation, Transmission Distribution, vol. 10, no. 14, pp. 3654-3662, 2016.

[22] Z. Yang, A. Bose, H. Zhong, N. Zhang, Q. Xia, and C. Kang, "Optimal reactive power dispatch with accurately modeled discrete control devices: A successive linear approximation approach," IEEE Transactions on Power Systems, vol. 32, no. 3, pp. 2435-2444, 2017.

[23] P. Fortenbacher and T. Demiray, "Linear/quadratic programmingbased optimal power flow using linear power flow and absolute loss approximations," International Journal of Electrical Power \& Energy Systems, vol. 107, pp. 680 - 689, 2019. [Online]. Available: http://www.sciencedirect.com/science/article/pii/S0142061518325377

[24] A. A. Mohamed and B. Venkatesh, "Line-wise optimal power flow using successive linear optimization technique," IEEE Transactions on Power Systems, vol. 34, no. 3, pp. 2083-2092, 2019.

[25] L. Sampath, B. V. Patil, H. Gooi, J. Maciejowski, and K. Ling, "A trust-region based sequential linear programming approach for ac optimal power flow problems," Electric Power Systems Research, vol. 165, pp. 134 - 143, 2018. [Online]. Available: http://www.sciencedirect. com/science/article/pii/S0378779618302955

[26] R. Byrd, N. Gould, J. Nocedal, and R. Waltz, "An algorithm for nonlinear optimization using linear programming and equality constrained subproblems," Mathematical Programming, vol. 100, no. 1, pp. 27-48, 2004, cited By 70.

[27] S. Mhanna and P. Mancarella, "An exact sequential linear programming algorithm for the optimal power flow problem," IEEE Transactions on Power Systems, pp. 1-1, 2021.

[28] A. Castillo, P. Lipka, J. P. Watson, S. S. Oren, and R. P. O’Neill, "A successive linear programming approach to solving the IV-ACOPF," IEEE Transactions on Power Systems, vol. 31, no. 4, pp. 2752-2763, July 2016.

[29] A. Wahid, S. A. Sadat, M. Ardakani, and A. Tajalli, "Power system emulator based on pll architecture," in 2020 IEEE International Symposium on Circuits and Systems (ISCAS), 2020, pp. 1-4.

[30] S. A. Sadat, "Evaluating the Performance of Various ACOPF Formulations Using Nonlinear Interior-Point Method," IEEE International Smart Cities Conference (ISC2), 82021.

[31] S. A. Sadat and K. Kim, "Numerical performance of different formulations for alternating current optimal power flow," preprint, 2021.

[32] ARPAE Grid Optimization Competition, "SCOPF Problem Formulation: Challenge 1," 2019, p. 18. [Online]. Available: https://gocompetition.energy.gov/sites/default/files/SCOPF_ Problem_Formulation_Challenge_1_20190412.pdf

[33] J. Nocedal and S. Wright, "Numerical optimization. 2nd edn springer," New York, 2006.

[34] S. A. Sadat, X. Rui, and M. Sahraei-Ardakani, "Computational Impacts of SVCs on Optimal Power Flow using Sequential Quadratic Programming and Approximated Active-Sets," preprint, 2021.

[35] The U.S. Department of Energy, "Grid Optimization (GO) competition - Challenge I," 2019. [Online]. Available: https://gocompetition.energy. gov/challenges/challenge- 1

[36] K. Kim, C. G. Petra, and V. M. Zavala, "An asynchronous bundletrust-region method for dual decomposition of stochastic mixed-integer programming," SIAM Journal on Optimization, vol. 29, no. 1, 12019. [Online]. Available: https://www.osti.gov/biblio/1497321 